\title{
A Nonuniform Matroid Which Violates the Isotopy Conjecture
}

\author{
Neil L. White \\ Department of Mathematics, University of Florida, Gainesville, FL 32611, USA
}

\begin{abstract}
The Isotopy Conjecture for Oriented Matroids states that the realization space over the real number field of an oriented matroid is path-connected. We provide a nonuniform counterexample of rank 3 on 42 points.
\end{abstract}

An oriented matroid $M(S)$ of rank $k$ is a set $S$ together with a function $\theta: S^{k} \rightarrow$ $\{0,1,-1\}$ satisfying certain axioms [2], [1]. The precise axioms need not concern us here, for we will be dealing with real-represented oriented matroids, that is, where we are given a function $f: S \rightarrow \mathbb{R}^{k}$, which by defining $\theta\left(s_{1}, \ldots s_{k}\right)=$ $\operatorname{sgn}\left(\operatorname{det}\left(f\left(s_{1}\right), \ldots, f\left(s_{k}\right)\right)\right) \in\{0,1,-1\}$ gives an oriented matroid. An oriented matroid is said to be uniform if $\theta$ does not take the value 0 on $k$-tuples of distinct elements.

If $S$ is a finite set of cardinality $n$, then a real-representation of $M(S)$ may be thought of as a vector in $\mathbb{R}^{n k}$, and we may ask whether the set of all real-realizations of $M(S)$ is connected in the usual topology of $\mathbb{R}^{n k}$. That this should be so for all realizable oriented matroids is the Isotopy Conjecture for Oriented Matroids, due to Ringel [4], and considered by Goodman and Pollack [3], Bokowski and Sturmfels [2], and Sturmfels [5]. The conjecture is known to be true for uniform rank-3 oriented matroids of up to nine points, and for oriented matroids admitting a reduction sequence, while failing for partial oriented matroids (where the signs are left unspecified for some $k$-tuples) already for small examples [5]. We now provide a counterexample to the conjecture consisting of a rank-3 oriented matroid on 42 points. This example is highly nonuniform, and leaves the conjecture restricted to uniform oriented matroids intact.

The idea of the counterexample is quite simple. We consider a rank-3 matroid which requires one of two roots of a quadratic polynomial to be used in any realization over the reals in projective canonical form, and is otherwise projectively uniquely realizable. Such a matroid may be constructed by well-known techniques of geometric addition and multiplication (see Chapter 1.7 in [6]). This gives two real realizations with the property that every real realization of 
Table 1

\begin{tabular}{|c|c|c|c|c|c|c|c|c|c|c|c|c|c|c|c|c|c|c|c|}
\hline 1 & 2 & 3 & 4 & 5 & 6 & 7 & 8 & 9 & 10 & 11 & 12 & 13 & 14 & 15 & 16 & 17 & 18 & 19 & 20 \\
\hline 1 & 0 & 0 & 1 & 1 & 1 & 1 & 0 & 0 & 1 & 1 & 1 & 1 & 0 & 1 & 1 & 1 & 1 & 1 & 1 \\
\hline 0 & 1 & 0 & 1 & 0 & 1 & 0 & 1 & 1 & -1 & 0 & 1 & 0 & 1 & 3 & 0 & 9 & 0 & 27 & 0 \\
\hline 0 & 0 & 1 & 1 & $r$ & 0 & 1 & 1 & -1 & 0 & 2 & 2 & 3 & -3 & 0 & 9 & 0 & 27 & 0 & 81 \\
\hline \multicolumn{2}{|c|}{21} & \multicolumn{2}{|l|}{22} & \multicolumn{2}{|l|}{23} & 24 & \multicolumn{2}{|r|}{25} & 26 & \multicolumn{2}{|c|}{27} & \multicolumn{2}{|l|}{28} & 29 & 30 & \multicolumn{2}{|c|}{31} & \multicolumn{2}{|c|}{32} \\
\hline \multicolumn{2}{|c|}{1} & \multicolumn{2}{|l|}{0} & \multicolumn{2}{|c|}{1} & 1 & & 0 & 1 & & 1 & & 1 & 0 & \multicolumn{2}{|r|}{1} & \multicolumn{2}{|c|}{1} \\
\hline \multicolumn{2}{|c|}{81} & \multicolumn{2}{|l|}{ 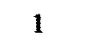 } & \multicolumn{2}{|c|}{0} & 1 & 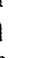 & 1 & 0 & & 1 & 0 & 3 & & 1 & \multicolumn{2}{|c|}{0} & \multicolumn{2}{|c|}{1} \\
\hline \multicolumn{2}{|c|}{0} & \multicolumn{2}{|l|}{-27} & \multicolumn{2}{|c|}{2187} & 2187 & & 27 & 2160 & \multicolumn{2}{|c|}{2160} & \multicolumn{2}{|c|}{2161} & $r$ & 81 & \multicolumn{2}{|c|}{$r-81$} & & -81 \\
\hline & 33 & & 34 & & 35 & & 36 & & 37 & 38 & & 39 & & 40 & & 41 & & 42 & \\
\hline & 0 & & 1 & & 1 & & & 1 & 0 & 1 & & 0 & & 1 & & 1 & & 0 & \\
\hline & 1 & & $r$ & & 0 & & & 1 & 1 & 0 & & 1 & & 0 & & 1 & & 1 & \\
\hline & $r+81$ & & 0 & & $r^{2}-8$ & & -3 & 3 & 9 & -12 & & -12 & & $12 r$ & & $12 r$ & & $r^{2}-8$ & \\
\hline
\end{tabular}

the particular matroid must be projectively equivalent to one of the two. Hence the realization space of the matroid has two components. The difficulty is in finding a particular quadratic polynomial and choosing a particular matroid with only two such realizations up to projective equivalence, so that all of the nonzero determinants take the same sign in both realizations. We choose the polynomial $x^{2}-93 x+2161$, which has roots approximately equal to 47.618 and 45.382 . In the representation in Table 1, we let $r$ denote either of these roots. The projective uniqueness of the two realizations follows from the fact that every point after the first five points is the intersection of two lines determined by proceeding points. The requirement that $r$ be a root of the above polynomial is simply the specification that the determinant of points 28,41 , and 42 is zero. The proof is then completed by exhaustive verification, accomplished on a personal computer, that every determinant is positive, negative, or zero in one realization if and only if it is positive, negative, or zero (resp.) in the other realization.

\section{References}

1. R. Bland and M. Las Vergnas, Orientability of matroids, $J$. Combin. Theory Ser, B 24 (1978), 94-123.

2. J. Bokowski and B. Sturmfels, On the coordinatization of oriented matroids, Discrete Comput. Geom. 1 (1986), 293-306.

3. J. E. Goodman and R. Pollack, Tagungsbericht Oberwolfach, Tagung über kombinatorische Geometrie, September 1984, List of problems.

4. G. Ringel, Teilungen der Ebene durch Geraden oder topologische Geraden, Math. Z. 64 (1956), $79-102$.

5. B. Sturmfels, Computational synthetic geometry, Ph.D. thesis, University of Washington, Seattle, WA, 1987.

6. N. White, ed., Combinatorial Geometries, Cambridge University Press, Cambridge, 1987.

Received June 29, 1987.

Note added in proof. Jaggi and Mani-Levitska have recently found a uniform counterexample on 17 points. 